\title{
Optimal Surveillance Strategies After Surgery for Renal Cell Carcinoma
}

\author{
Mark D. Tyson, MD, and Sam S. Chang, MD, MBA
}

\section{Abstract}

One in 5 patients who undergo surgical resection for clinically localized renal cell carcinoma (RCC) develop local and/or distant recurrences which, when detected early, may be amenable to salvage local and systemic therapies. When considering that approximately half of these recurrences will occur during the first 2 years, a clear rationale exists for optimizing surveillance strategies after surgery. Although there is a notable dearth of high-quality data on this subject, clinical principles can guide clinicians as they attempt to balance the burden of surveillance strategies with potential clinical benefit. The objective of this review is to summarize the evidence regarding optimal surveillance protocols after surgery for RCC. We provide an overview of the rationale supporting surveillance after surgery, a summary of the American Urological Association and NCCN guidelines, reasons against routine long-term surveillance, surveillance costs, and ancillary issues, such as the utility of bone scan, PET/CT scan, and surveillance after thermoablation.

J Natl Compr Canc Netw 2017;15(6):835-840 doi:10.6004/jnccn.2017.0102

The incidence of renal cell carcinoma (RCC) has been steadily increasing for decades. ${ }^{1}$ The mainstay of treatment for clinically localized disease is radical nephrectomy and, when technically feasible, partial nephrectomy. Yet, up to $20 \%$ to $30 \%$ of patients who undergo surgical resection for clinically localized tumors may develop local and/or distant recurrences, which, when detected early, may be amenable to salvage local and systemic therapies. ${ }^{2-4}$ When considering that approximately half of these recurrences will occur within the first 2 years, ${ }^{5}$ a clear rationale exists for optimizing surveillance strategies for patients who have undergone surgical resection for clinically localized RCC.

Treating physicians, however, disagree on what constitutes an "optimal" strategy. ${ }^{6}$ This lack of consensus is, in no small part, due to the variable nature of the timing and anatomic location of RCC recurrence and the lack of empirical data supporting the beneficial impact of early salvage therapies. ${ }^{7}$ Although most recurrences present within the first couple of years after surgery and approxi-

From the Department of Urologic Surgery, Vanderbilt University Medical Center, Nashville, Tennessee.

Submitted December 1, 2016; accepted for publication March 6, 2017.

The authors have disclosed that they have no financial interests,

arrangements, affiliations, or commercial interests with the

manufacturers of any products discussed in this article or their mately half of all distant recurrences occur in the lung, late recurrences and nonpulmonary sites of metastatic disease are not uncommon. ${ }^{5,8-11} \mathrm{~A}$ small percentage of patients will develop recurrences $\geq 10$ years after surgical therapy, with some presenting with distant recurrences $>20$ years later. ${ }^{11,12}$ Yet, subjecting every patient to $\geq 20$ years of surveillance is certainly not optimal given the manifestly high cost and risk associated with continued surveillance imaging over time. ${ }^{13}$ However, by the same token, the absence of evidence does not justify forgoing surveillance altogether, as highlighted by the American Urological Association (AUA) and NCCN guidelines, which attempt to balance the burden of surveillance strategies with potential clinical benefit.

In this context, we aim to summarize the evidence regarding the optimal surveillance protocols after surgery for RCC. We provide an overview of the rationale supporting surveillance after surgery, a summary of the AUA and NCCN guidelines, reasons against routine long-term surveillance, surveillance costs, and ancillary

competitors. This work was in part supported by NCI Grant 5T32CA106183 (M.D.T.).

Correspondence: Mark D. Tyson, MD, Vanderbilt University Medical Center, Department of Urologic Surgery, A1302 Medical Center North, Nashville, TN 37232-2765.

E-mail: mark.tyson@vanderbilt.edu 
issues, such as the utility of bone scan, PET/CT scan, and surveillance after thermoablation.

\section{Summary of NCCN Guidelines}

For patients with stage I RCC (T1a and T1b), a history and physical examination (H\&P) with a comprehensive metabolic panel is recommended every 6 months for the first 2 years, then annually up to 5 years. ${ }^{2}$ If the patient underwent a partial nephrectomy, a baseline abdominal CT, MRI, or ultrasound is recommended within 3 to 12 months of surgery, then annually for 3 years if the baseline scan is negative. Recognizing that the risk of visualizing a local recurrence, even in the setting of a positive margin, is low within the first few months after surgery, the rationale for performing a baseline CT or MRI is that the kidney architecture can be substantially altered after a partial nephrectomy, and baseline imaging serves as a comparison point for future surveillance studies. This may be even more important in the era of Hem-o-lok clips, which can confound the appearance of the post-partial nephrectomy renorrhaphy bed. If the patient underwent a radical nephrectomy, a baseline abdominal CT, MRI, or ultrasound should be performed within 3 to 12 months, but imaging beyond that is optional if the baseline scan is negative. Chest imaging (radiograph or CT) is recommended annually during the first 3 years, then as clinically indicated thereafter. Brain, pelvis, and bone imaging is only recommended if clinically indicated.

Although NCCN does not make formal recommendations regarding routine surveillance beyond the 5-year period, the guidelines state that surveillance may be extended beyond 5 years at the physician's discretion. There may be several reasons why a clinician would choose to continue surveillance for selected patients who have undergone a partial nephrectomy for low-risk disease. First, some patients, who are by definition stage I, have highrisk characteristics that make them more prone to local and distant recurrences (eg, positive margins, high Fuhrman grade, aberrant histology, pathologic upgrading). Furthermore, with increasing attention on the sequela of chronic kidney disease, there has been an impressive expansion in the use of partial nephrectomy for treating clinical T1b tumors. Although these patients technically fall within the stage I designation, most would agree that they are at high risk of recurrence compared with those with pT1a tumors. Lastly, metachronous renal tumors in either the ipsilateral or contralateral kidney are found in as many as $20 \%$ of patients with papillary RCC or familial RCC. ${ }^{14}$ Taken together, a strong case could be made for continuing surveillance up to and perhaps even beyond the 5-year period for patients with clinical risk factors for recurrence.

For patients who have undergone radical nephrectomy for stage II or III disease, an H\&P is recommended every 3 to 6 months through the third year, then annually through the fifth year. Comprehensive metabolic panels should be ordered every 6 months for the first 2 years, then annually through year 5. A baseline abdominal CT or MRI is recommended within 3 to 6 months, then at 3-to 6 -month intervals for the first 3 years, followed by an annual schedule until year 5 . It should be noted that an ultrasound is a category $2 \mathrm{~B}$ option for stage III disease in the NCCN Guidelines. ${ }^{2}$ A chest CT is recommended within 3 to 6 months after surgery, every 3 to 6 months thereafter through year 3 , then annually through year 5 if the baseline imaging is negative. Brain, pelvis, and bone imaging is only recommended if clinically indicated.

\section{Summary of AUA Guidelines}

For patients with low-risk disease, which is defined as $\mathrm{T} 1 \mathrm{NO} / \mathrm{X}$ and for whom the risk of recurrence is approximately $1 \%$ to $8 \%,{ }^{15,16}$ the AUA recommends a H\&P at 6, 12, 24, and 36 months, with further follow-up performed at the discretion of the treating physician. ${ }^{17}$ Bloodwork, such as serum creatinine levels, and urine tests are performed as clinically indicated. For patients who have undergone a partial nephrectomy, abdominal imaging (either an MRI or CT) is recommended between 3 and 12 months after surgery. If this initial postoperative scan is negative, annual abdominal imaging (ultrasound, CT, MRI) is optional up to 3 years.

For patients who underwent a radical nephrectomy, an abdominal ultrasound is an acceptable substitute for abdominal CT or MRI, but 1 of these 3 studies should be performed within 3 to 12 months postsurgery. Because the risk of local or distant recurrence is so low in patients without familial RCC (2\%-4\%), abdominal imaging after this initial study is optional. ${ }^{18-22}$ With respect to chest surveillance, 
either a chest radiograph or CT is recommended annually for 3 years, then as clinically indicated. Because the clinical suspicion for pulmonary metastatic disease is so low for most low-risk patients, a chest radiograph is sufficient for most patients undergoing surveillance after nephrectomy or partial nephrectomy. If the clinical suspicion is higher on account of high-risk features mentioned earlier, then a chest CT may be indicated. At this time, routine surveillance of the brain, pelvis, or bone is not recommended unless clinically indicated.

For patients with moderate- to high-risk disease (defined as T2-4NO/X or TanyN1 disease), the risk of local and distant recurrences may be much higher (30\%-78\%), which necessitates a more intense surveillance protocol. ${ }^{17}$ In addition to a biannual $H \& P$ for 5 years, a baseline CT or MRI of the abdomen and chest is recommended within 3 to 6 months, then every 6 months until 5 years. It should be noted that if the initial abdominal imaging is negative, the clinician could consider obtaining abdominal ultrasounds as opposed to CT every 6 months. This more intense surveillance is predicated on the assumption that most of these patients will be amenable to salvage therapy in the event of a recurrence and that earlier detection of this recurrence will lead to improved outcomes; a belief that is admittedly lacking in evidentiary support.

\section{Years and Beyond}

Although the AUA and NCCN discuss imaging up to 5 years for moderate- to high-risk patients, the clinician, for reasons enumerated earlier, may wish to continue with surveillance beyond this 5-year period for select patients at higher risk of recurrence. However, a closer look at the evidence regarding long-term intense surveillance casts serious doubts on the utility of this strategy for every patient. First, late metastatic recurrences have been reported to occur in only $1.8 \%$ of patients after 5 years. ${ }^{11}$ In a report of $>2,368$ patients who underwent surgery for clinically localized disease, only 44 experienced a recurrence 5 years after nephrectomy. ${ }^{11}$ Although only $25 \%$ of these patients were symptomatic at presentation, the median survival after diagnosis of recurrent disease was approximately 6 years in all patients. When stratified by symptoms, patients who were symptomatic experienced a substantially lower median survival (3 years).
In a separate analysis of 470 patients who underwent curative surgical resection for RCC and had not developed a recurrence within 10 years, only 30 developed a recurrence and approximately $64 \%$ of those patients were alive at 20 years compared with $78 \%$ of patients whose disease did not recur. ${ }^{12}$ Other studies suggest that recurrences can occur as late as 30 to 40 years later. ${ }^{23,24}$ However, these low rates of recurrence, reasonably good survival functions, and isolated case reports of extremely late recurrences hardly constitute sufficient justification for subjecting the remainder of the patients to the burdensome cost and potentially dangerous radiation exposure of annual surveillance. ${ }^{25}$ In other words, what is good for an individual patient in retrospect may not necessarily be good for the rest of the population moving forward.

\section{Laboratory Testing}

The timely detection of progressive renal deterioration after nephrectomy permits an early referral to nephrology in an effort to limit any further progression in kidney disease. Both NCCN and the AUA recommend routine laboratory testing, such as a comprehensive metabolic panel and other tests as indicated; lactate dehydrogenase $(\mathrm{LDH})$ is included in many prediction models because it improves prognostication, particularly for patients with advanced disease. ${ }^{26,27}$ Although few empirical data exist supporting the routine use of $\mathrm{LDH}$ in the surveillance of patients who have undergone nephrectomy for clinically localized disease, the AUA notes that it may have a role as a measure of general organ function. Alkaline phosphatase (ALP) is yet another potential prognostic marker for patients with advanced disease. ${ }^{28}$ When limited to nonmetastatic tumors only, multivariable analysis has demonstrated that ALP is an independent predictor of disease-specific survival. However, several retrospective analyses have challenged the notion that ALP is useful in the follow-up of asymptomatic patients with RCC..$^{29,30}$

\section{Bone Scan}

Routine use of bone scan in the follow-up of asymptomatic patients with nonmetastatic RCC and normal ALP levels is not supported in the literature. Emerging evidence suggests that the probability of finding bony metastatic disease in the absence of an elevated ALP 
or clinical symptoms is $<1 \% .{ }^{31,32}$ However, in the presence of an elevated ALP or clinical symptoms that are worrisome for bony metastatic disease, the probability of a positive bone scan increases to approximately $5 \%$ to $10 \%$. ${ }^{33}$ Taken together, radionuclide bone scans should not be a routine part of surveillance strategies postnephrectomy in most patients, but rather driven by clinical symptoms and serum ALP values.

\section{Brain Imaging}

The routine use of head MRI or CT for surveillance after nephrectomy is not recommended due to an exceedingly low probability of finding metastatic disease in this population. However, in patients with neurologic signs or symptoms, prompt neurologic evaluation with cross-sectional imaging of the head is recommended. MRI may have better sensitivity than a CT scan for detecting small central nervous system metastatic deposits, but CT may be more appropriate in the setting of acute neurologic decompensation requiring immediate treatment. ${ }^{34}$

\section{PET Scan and Molecular Markers}

There is insufficient evidence to support the use of molecular markers, such as ki-67, p53, and vascular endothelial growth factor, in the postnephrectomy risk stratification of patients with nonmetastatic RCC. ${ }^{17}$ Similarly, given the lack of empirical evidence regarding the sensitivity and specificity of PET/CT scan in detecting local or distant recurrences in patients undergoing surveillance after nephrectomy, its use is currently discouraged. ${ }^{17}$

\section{Surveillance After Thermoablation}

Thermoablative techniques are increasingly being used as alternative approaches for the management of clinical T1 RCC. Although the focus of this article is surveillance after surgery, 3 points regarding surveillance after thermoablation are worthy of brief discussion. First, although the risk of developing distant metastatic disease after thermoablation appears to be comparable to nephrectomy, local recurrences are much more likely. ${ }^{35,36}$ As a result, closer attention must be paid to monitoring the ablated kidney for local recurrences, especially because tumor recurrences can be easily re-treated with thermoabla- tive techniques. A posttreatment biopsy should be performed if there is any progressive increase in the size of an ablated lesion, with or without contrast enhancement; new concerning areas around the treated zone; or failure of the treated lesion to regress in size over time. ${ }^{17}$ Second, Weight et $\mathrm{al}^{37}$ advocate these patients be followed similar to intermediateto high-risk postsurgical patients, and many advocate for a biopsy at 6 months given the poor correlation between imaging and pathology. However, NCCN recommends a postprocedural biopsy if there are clinical indications, such as new enhancement, progressive enlargement, new nodularity, failure to regress, or satellite or port site lesions. ${ }^{2}$ Lastly, patients undergoing thermoablation should have a pretreatment biopsy. If the results are consistent with a benign histology and there is radiographic confirmation of treatment success with no evidence of treatment-related complications, surveillance imaging can be safely avoided.

\section{Cost}

No discussion regarding the optimal surveillance strategy for RCC would be complete without briefly discussing the costs associated with surveillance. Predicted costs associated with 5 years of surveillance after a partial nephrectomy based on the AUA/NCCN guidelines is roughly $\$ 3,904 \mathrm{USD} .^{38} \mathrm{~A}$ separate study showed that in order to capture $95 \%$ of all recurrences up to 15 years, the costs associated with surveillance increase to $\$ 9,856$ USD per patient. ${ }^{13}$ Future research is needed to identify optimal surveillance strategies that maximize early detection of recurrent $\mathrm{RCC}$ while minimizing costs and radiation exposure.

\section{Future Directions}

What is perhaps most striking about the state of the current evidence is the relative lack of high-quality studies that precisely estimate the effect of surveillance imaging on important clinical variables, such as overall survival, disease-specific survival, and quality of life. Although observational and retrospective studies are useful for understanding how surveillance strategies may impact long-term survival, they are ill-equipped for strong causal inferences. As a result, long-term, prospective, preplanned, observational studies or randomized trials are still needed to firmly 
answer the many remaining questions, such as does early detection significantly improve survival? Does intense surveillance offer any advantages over routine surveillance? What defines treatment failure after thermoablation? Is there a role for tumor or tissue markers in predicting survival, recurrence, or metabolic sequelae? What is the effect of adjuvant systemic therapy for surveillance-detected recurrences? What are the long-term effects of cumulative radiation exposure posed by intense surveillance? These questions are among many that still need to be answered before strong statements can be endorsed by existing guideline bodies.

\section{Conclusions}

The optimal surveillance strategy for patients who have undergone surgical resection for clinically localized RCC remains unknown. However, because most recurrences occur within the first few years after surgery, optimal surveillance strategies undoubtedly focus on surveillance during this period. However, routine long-term surveillance, namely beyond 5 years, is not directly supported by existing data, although there may be a rationale for long-term surveillance for patients with high-risk characteristics, such as positive margins, aberrant histology, familial RCC, young age, stage $\geq \mathrm{T} 3$, or papillary RCC.

\section{References}

1. Tyson MD, Humphreys MR, Parker AS, et al. Age-period-cohort analysis of renal cell carcinoma in United States adults. Urology 2013;82:43-47.

2. Motzer RJ, Jonasch E, Agarwal N, et al. NCCN Clinical Practice Guidelines in Oncology: Kidney Cancer. Version 2.2017. Accessed April 17,2017 . To view the most recent version of these guidelines, visit NCCN. org.

3. Leibovich BC, Blute ML, Cheville JC, et al. Prediction of progression after radical nephrectomy for patients with clear cell renal cell carcinoma: a stratification tool for prospective clinical trials. Cancer 2003;97:16631671.

4. Cindolo L, Patard JJ, Chiodini P, et al. Comparison of predictive accuracy of four prognostic models for nonmetastatic renal cell carcinoma after nephrectomy: a multicenter European study. Cancer 2005;104:1362-1371.

5. Eggener SE, Yossepowitch O, Pettus JA, et al. Renal cell carcinoma recurrence after nephrectomy for localized disease: predicting survival from time of recurrence. J Clin Oncol 2006;24:3101-3106.

6. Sohn W, Graves AJ, Tyson MD, et al. An empiric evaluation of the effect of variation in intensity of followup for surgically treated renal neoplasms on cancer specific survival. J Urol 2017;197:37-43.

7. Brennan MF. Follow-up is valuable and effective: true, true and unrelated? Ann Surg Oncol 2000;7:2-3.

8. Levy DA, Slaton JW, Swanson DA, Dinney CP. Stage specific guidelines for surveillance after radical nephrectomy for local renal cell carcinoma. J Urol 1998;159:1163-1167.

9. Hafez KS, Novick AC, Campbell SC. Patterns of tumor recurrence and guidelines for followup after nephron sparing surgery for sporadic renal cell carcinoma. J Urol 1997;157:2067-2070.

10. Ljungberg B, Alamdari FI, Rasmuson T, Roos G. Follow-up guidelines for nonmetastatic renal cell carcinoma based on the occurrence of metastases after radical nephrectomy. BJU Int 1999;84:405-411.

11. Adamy A, Chong KT, Chade D, et al. Clinical characteristics and outcomes of patients with recurrence 5 years after nephrectomy for localized renal cell carcinoma. J Urol 2011;185:433-438.

12. Miyao N, Naito $S$, Ozono S, et al. Late recurrence of renal cell carcinoma: retrospective and collaborative study of the Japanese Society of Renal Cancer. Urology 2011;77:379-384.

13. Stewart SB, Thompson RH, Psutka SP, et al. Evaluation of the National Comprehensive Cancer Network and American Urological Association renal cell carcinoma surveillance guidelines. J Clin Oncol 2014;32:4059_ 4065.

14. Richstone L, Scherr DS, Reuter VR, et al. Multifocal renal cortical tumors: frequency, associated clinicopathological features and impact on survival. J Urol 2004;171:615-620.

15. Herr HW. Partial nephrectomy for incidental renal cell carcinoma. Br J Urol 1994;74:431-433.

16. Gill IS, Kavoussi LR, Lane BR, et al. Comparison of 1,800 laparoscopic and open partial nephrectomies for single renal tumors. J Urol 2007;178:41-46.
17. Donat SM, Diaz M, Bishoff JT, et al. Follow-up for clinically localized renal neoplasms: AUA guideline. J Urol 2013;190:407-416.

18. Antonelli A, Cozzoli A, Zani D, et al. The follow-up management of nonmetastatic renal cell carcinoma: definition of a surveillance protocol. BJU Int 2007;99:296-300.

19. Becker F, Siemer S, Tzavaras A, et al. Long-term survival in bilateral renal cell carcinoma: a retrospective single-institutional analysis of 101 patients after surgical treatment. Urology 2008;72:349-353.

20. Wiklund F, Tretli S, Choueiri TK, et al. Risk of bilateral renal cell cancer. J Clin Oncol 2009;27:3737-3741.

21. Stern JM, Svatek R, Park $S$, et al. Intermediate comparison of partial nephrectomy and radiofrequency ablation for clinical T1a renal tumours. BJU Int 2007;100:287-290.

22. Klatte T, Patard JJ, Wunderlich $\mathrm{H}$, et al. Metachronous bilateral renal cell carcinoma: risk assessment, prognosis and relevance of the primary-free interval. J Urol 2007;177:2081-2086; discussion 2086-2087.

23. Featherstone JM, Bass P, Cumming J, Smart CJ. Solitary, late metastatic recurrence of renal cell carcinoma: two extraordinary cases. Int J Urol 2006;13:1525-1527.

24. Tapper $H$, Klein $H$, Rubenstein W, et al. Recurrent renal cell carcinoma after 45 years. Clin Imaging 1997;21:273-275.

25. Fry RJ, Grosovsky A, Hanawalt PC, et al. The impact of biology on risk assessment--workshop of the National Research Council's Board on Radiation Effects Research. July 21-22, 1997, National Academy of Sciences, Washington, DC. Radiat Res 1998;150:695-705.

26. Heng DY, Xie W, Regan MM, et al. Prognostic factors for overall survival in patients with metastatic renal cell carcinoma treated with vascular endothelial growth factor-targeted agents: results from a large, multicenter study. J Clin Oncol 2009;27:5794-5799.

27. Motzer RJ, Bacik J, Mariani T, et al. Treatment outcome and survival associated with metastatic renal cell carcinoma of non-clear-cell histology. J Clin Oncol 2002;20:2376-2381.

28. Lee SE, Byun SS, Han JH, et al. Prognostic significance of common preoperative laboratory variables in clear cell renal cell carcinoma. BJU Int 2006;98:1228-1232.

29. Bos SD, Piers DA, Mensink HJ. Routine bone scan and serum alkaline phosphatase for staging in patients with renal cell carcinoma is not costeffective. Eur J Cancer 1995;31A:2422-2423.

30. Kriteman L, Sanders WH. Normal alkaline phosphatase levels in patients with bone metastases due to renal cell carcinoma. Urology 1998;51:397399.

31. Blacher E, Johnson DE, Haynie TP. Value of routine radionuclide bone scans in renal cell carcinoma. Urology 1985;26:432-434.

32. Lindner A, Goldman DG, deKernion JB. Cost effective analysis of prenephrectomy radioisotope scans in renal cell carcinoma. Urology 1983;22:127-129.

33. Koga $S$, Tsuda $S$, Nishikido $M$, et al. The diagnostic value of bone scan in patients with renal cell carcinoma. J Urol 2001;166:2126-2128. 
34. Young RJ, Sills AK, Brem S, Knopp EA. Neuroimaging of metastatic brain disease. Neurosurgery 2005;57:S10-23; discussion S1-4.

35. Kunkle DA, Egleston BL, Uzzo RG. Excise, ablate or observe: the small renal mass dilemma—a meta-analysis and review. J Urol 2008;179:12271233; discussion 1233-1234.

36. Klatte $\mathrm{T}$, Grubmüller B, Waldert M, et al. Laparoscopic cryoablation versus partial nephrectomy for the treatment of small renal masses: systematic review and cumulative analysis of observational studies. Eur Urol 2011;60:435-443.

37. Weight CJ, Kaouk JH, Hegarty NJ, et al. Correlation of radiographic imaging and histopathology following cryoablation and radio frequency ablation for renal tumors. J Urol 2008;179:1277-1281; discussion 1281-1283.

38. Lobo JM, Nelson M, Nandanan N, Krupski TL. Comparison of renal cell carcinoma surveillance guidelines: competing trade-offs. J Urol 2016;195:1664-1670.

Recorded Presentations from the

$\mathrm{NCCN}$
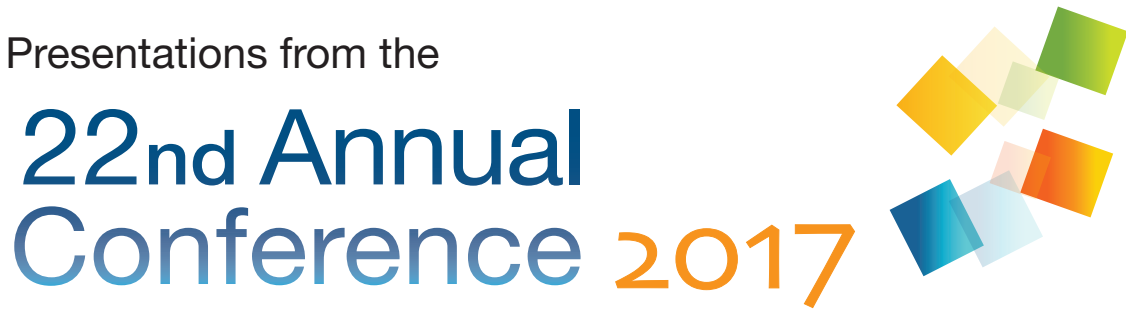

IMPROVING THE QUALITY, EFFECTIVENESS, AND EFFICIENCY OF CANCER CARE ${ }^{\text {TM }}$

Earn CME/CE credits online! More than 20 educational sessions featuring the latest advances in oncology care.

\section{education.nccn.org/ac2017}

This enduring material is approved for AMA PRA Category 1 Creditit for physicians and will award contact hours for nurses, pharmacists, and other health care professionals. Complete accreditation information is available online.

This activity is supported by educational grants from Ariad; Astellas and Medivation, Inc.; AstraZeneca; BTG International Inc.; Celgene Corporation;

Celldex Therapeutics; Foundation Medicine; Genentech; Genomic Health, Inc.; Gilead Sciences, Medical Affairs; Lilly; Merrimack Pharmaceuticals, Inc.;

Novartis; Pfizer; Prometheus Laboratories Inc.; Taiho Oncology; and Takeda Oncology. This activity is supported by independent educational grants from

This educational activity is supported by a medical education grant from Exelixis, Inc. 